\title{
The physics and the structure of the quasar-driven outflow in Mrk 231
}

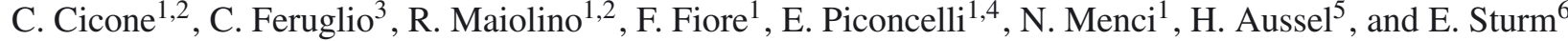 \\ ${ }^{1}$ Cavendish Laboratory, University of Cambridge 19 J. J. Thomson Avenue, Cambridge CB3 OHE, UK \\ e-mail: c.cicone@mrao.cam.ac.uk \\ 2 INAF - Osservatorio Astronomico di Roma (OAR), via Frascati 33, 00040 Monteporzio Catone, Italy \\ 3 Institut de Radio Astronomie Millimétrique (IRAM), 300 rue de la Piscine, St. Martin d'Hères, France \\ 4 XMM-Newton Science Operations Centre, ESA, PO Box 78, 28691 Villanueva de la Cañada, Madrid, Spain \\ ${ }^{5}$ Laboratoire AIM, DSM/Irfu/Service d'Astrophysique, CEA Saclay, 91191 Gif-sur-Yvette, France \\ ${ }^{6}$ Max Planck Institute für Extraterrestrische Physik (MPE), Postfach 1312, 85741 Garching, Germany
}

Received 9 January 2012 / Accepted 22 May 2012

\begin{abstract}
Massive AGN-driven outflows are invoked by AGN-galaxy co-evolutionary models to suppress both star formation and black hole accretion. Massive molecular outflows have been discovered in some AGN hosts. However, the physical properties and structures of these AGN-driven molecular outflows are still poorly constrained. Here we present new IRAM PdBI observations of Mrk 231, the closest quasar known, targeting both the $\mathrm{CO}(1-0)$ and $\mathrm{CO}(2-1)$ transitions. We detect broad wings in both transitions, which trace a massive molecular outflow moving with velocities of up to $800 \mathrm{~km} \mathrm{~s}^{-1}$. The wings are spatially resolved at high significance levels $(5-11 \sigma)$, indicating that the molecular outflow extends to the kpc scale. The $\mathrm{CO}(2-1) / \mathrm{CO}(1-0)$ ratio of the red broad wings is consistent with the ratio observed in the narrow core, while the blue broad wing is less excited than the core. The latter result suggests that quasar-driven outflow models invoking shocks (which would predict higher gas excitation) are inappropriate for describing the bulk of the outflow in Mrk 231. However, we note that within the central $700 \mathrm{pc}$ the $\mathrm{CO}(2-1) / \mathrm{CO}(1-0)$ ratio of the red wing is slightly, but significantly, higher than in the line core, suggesting that shocks may play a role in the central region. We also find that the average size of the outflow anticorrelates with the critical density of the transition used as a wind tracer. This indicates that, although diffuse and dense clumps coexist in the outflowing gas, dense outflowing clouds have shorter lifetimes and that they evaporate into the diffuse component along the outflow or, more simply, that diffuse clouds are more efficiently accelerated to larger distances by radiation pressure.
\end{abstract}

Key words. galaxies: active - galaxies: evolution - galaxies: individual: Mrk 231 - quasars: general - radio lines: ISM ISM: molecules

\section{Introduction}

The ubiquitous discovery of "relic" supermassive black holes $(\mathrm{SMBH})$ in local bulges and of the tight correlations between their masses and bulge properties (e.g. Ferrarese \& Ford 2005; Marconi \& Hunt 2003; Gültekin et al. 2009, and references therein) indicates that nuclear activity likely plays an important role in galaxy evolution. Considerable attention has been drawn to powerful AGN-driven outflows as they may provide a very efficient mechanism enabling the nuclear activity occurring on sub-parsec scales to influence the surrounding host galaxy. The effect of these AGN winds on the interstellar matter (ISM) in the galaxy is often referred to as "AGN feedback". More specifically, AGN feedback is invoked by most current galaxy evolutionary models to explain the shortage of very massive galaxies in the local Universe, their red colors, and low gas content, as well as the $M_{\mathrm{BH}}-\sigma$ relation (e.g. Silk \& Rees 1998; Fabian 1999; King \& Pounds 2003; Fabian et al. 2006; Baldry et al. 2004; Granato et al. 2004; Di Matteo et al. 2005; Elvis 2006; Lapi et al. 2005; King 2005; Menci et al. 2006, 2008; Croton et al. 2006; Narayanan et al. 2008; Hopkins \& Elvis 2010).

There are two possible "flavors" of AGN feedback: feedback associated with the "radio-mode" (Croton et al. 2006) and feedback associated with the "quasar-mode". In this work, we focus only on the second one, which is the impulsive feedback exerted by AGNs during their bright active phases. One of the most common scenarios in theoretical models of quasar-mode feedback is that nuclear fast winds, generated by the AGN radiation pressure, deposit the energy produced by the nuclear activity into the ISM, compressing the ISM into a shock wave ("blast wave") that eventually blows the cold gas reservoir, available for both star formation and SMBH accretion, out of the host galaxy (Lapi et al. 2005; Menci et al. 2006, 2008; King 2010; Zubovas \& King 2012). Models generally assume that these outflows also contain a significantly large molecular component (Zubovas \& King 2012; Narayanan et al. 2008). If AGN-driven outflows can really expel large quantities of molecular gas (which constitutes the bulk of the ISM in the central regions and is the medium out of which stars form), then this would be an extremely effective mechanism for quenching star formation in massive galaxies.

Major observational breakthroughs in this field have been achieved by revealing massive and energetic quasar-driven outflows in several galaxies, both locally and at high redshift (Fischer et al. 2010; Feruglio et al. 2010; Rupke \& Veilleux 2011; Aalto et al. 2012; Sturm et al. 2011; Alatalo et al. 2011; Greene et al. 2011; Nesvadba et al. 2010, 2011; Alexander et al. 2010; Farrah et al. 2012; Cano-Díaz et al. 2012; Maiolino et al. 2012; see also the review by Fabian 2012). Within this context, Mrk 231, the closest quasar known, is the object in which a massive quasar-driven outflow was first discovered and that has been 
studied in the greatest detail (Fischer et al. 2010; Feruglio et al. 2010; Rupke \& Veilleux 2011; Aalto et al. 2012). Mrk 231 provides a unique, nearby laboratory for investigating AGN feedback, showing all of the typical features expected for a quasar transiting from the obscured, merger-driven and IR-luminous accretion phase, accompanied by strong circumnuclear star formation, to the unobscured, standard phase of a "blue" quasar (Lipari et al. 2009, and references therein), by expelling obscuring gas and dust. It is indeed the most luminous ultra-luminous infrared galaxy (ULIRG) in the local Universe (Sanders et al. 2003), with infrared (IR) luminosity $L_{\mathrm{IR}}(8-1000 \mu \mathrm{m})=1.33 \times 10^{46} \mathrm{erg} \mathrm{s}^{-1}$, and also hosts a very powerful $\left(L_{\mathrm{bol}, \mathrm{AGN}}=1.1 \times 10^{46} \mathrm{erg} \mathrm{s}^{-1}\right.$; e.g., Rupke \& Veilleux 2011), low-ionization broad absorption line (LoBAL) quasar (i.e. where the blue-shifted UV lines span velocities up to $\sim 8000 \mathrm{~km} \mathrm{~s}^{-1}$ ). Boroson \& Meyers (1992) argued that LoBAL quasars are young, heavily enshrouded AGNs, where the cocoon of gas and dust has a large covering factor, as supported by the results of Braito et al. (2004), who found that Mrk 231 is a very obscured X-ray source with a column density of $N_{\mathrm{H}} \approx 10^{24} \mathrm{~cm}^{-2}$. Mrk 231 also displays a distorted optical morphology, which implies that its host galaxy is in an advanced stage of a merging process (Lipari et al. 2009). The circumnuclear starburst in this source is very young ( $\$ 120 \mathrm{Myr})$, with an estimated star formation rate of $S F R \approx 200 M_{\odot} \mathrm{yr}^{-1}$ (Taylor et al. 1999; Davies et al. 2004).

A Herschel PACS spectrum of Mrk 231 has revealed a remarkably massive molecular outflow traced by prominent P-Cygni $\mathrm{OH}$ profiles at 65, 79, and $119 \mu \mathrm{m}$ with velocity shifts of $\sim 1000 \mathrm{~km} \mathrm{~s}^{-1}$ (Fischer et al. 2010). In Feruglio et al. (2010), we reported the detection of the molecular outflow based on IRAM PdBI observations, which revealed the broad $\left(F W Z I \sim 1500 \mathrm{~km} \mathrm{~s}^{-1}\right)$ wings of the $\mathrm{CO}(1-0)$ transition, that are marginally resolved with an extension of about $\sim 1 \mathrm{kpc}$, yielding an estimated mass-outflow rate of $\sim 700 M_{\odot} \mathrm{yr}^{-1}$, i.e., far higher than the ongoing SFR in Mrk 231. On the basis of kinetic power arguments, we also suggested that the molecular outflow is primarily driven by the quasar radiation pressure.

These findings were later confirmed by the analysis of the broad wings of the $\mathrm{H} \alpha$ and $\mathrm{Na}$ I D absorption line via IFU optical spectroscopy, which spatially resolved the outflow on the same scales as traced by the CO wings (Rupke \& Veilleux 2011). Furthermore, Aalto et al. (2012) also detected broad wings in the $\mathrm{HCN}(1-0), \mathrm{HCO}^{+}(1-0)$, and $\mathrm{HNC}(1-0)$ emission lines of Mrk 231, which are tracers of the high-density molecular gas, and confirmed the extent of the HCN(1-0) broad emission out to $0.7 \mathrm{kpc}$. They suggested that the molecular outflow in Mrk 231 is clumpy and dominated by the dense phase, and has an enhanced HCN abundance, which may indicate either that it contains a shocked medium in the outflow and/or a chemistry influenced by the AGN. Although these characteristics ensure that Mrk 231 is an exception in the local Universe, they are expected to be quite common at high redshift, where Mrk 231-like objects are thought to play a major role in the formation of present-day, red massive quiescent elliptical galaxies (Hopkins et al. 2008; Cattaneo et al. 2009). We note, in particular, that massive molecular outflows have been revealed in additional local ULIRGs through the detection of prominent $\mathrm{OH}$ P-Cygni profiles in their far-IR Herschel spectra (Sturm et al. 2011).

In this paper we present the analysis of a new set of IRAM PdBI observations of the $\mathrm{CO}(1-0)$ and $\mathrm{CO}(2-1)$ transitions in Mrk 231. The aim of this study is to improve the previous results achieved by Feruglio et al. (2010), by spatially resolving the molecular outflow with high significance $(5-11 \sigma)$. Moreover, we combine the $\mathrm{CO}(1-0)$ and $\mathrm{CO}(2-1)$ emission line data to investigate the physical conditions of the molecular outflowing gas in this unique source.

A $H_{0}=70.4 \mathrm{~km} \mathrm{~s}^{-1} \mathrm{Mpc}^{-1}, \Omega_{\mathrm{M}}=0.27, \Omega_{\Lambda}=0.73$ cosmology is adopted throughout this work.

\section{Observations}

Mrk 231 was observed in $\mathrm{CO}(1-0)$ and $\mathrm{CO}(2-1)$ with the IRAM millimeter-wave interferometer on Plateau de Bure (IRAM PdBI) between June 2009 and November 2010. The CO(2-1) observations presented allow us to investigate the broad wings in Mrk 231 for the first time in this transition. The new $\mathrm{CO}(1-0)$ instead allows us to improve on the previous Feruglio et al. (2010) observation by significantly increasing the signal on long baselines and, therefore, allowing us to investigate in more detail the extension of the outflow in this transition. Table 1 provides a technical description of these observations: the $\mathrm{CO}$ transition and the corresponding redshifted frequency $(z=0.04217)$, the dates of the observations, the array configurations and the number of antennas used, the on-source integration times, the beam sizes, and the relevant references.

The data were calibrated by using the CLIC software of the GILDAS package ${ }^{1}$. The main flux calibrator at $110.697 \mathrm{GHz}$ was MWC 349, whose flux at this frequency is $1.27 \mathrm{Jy}$; the principal flux calibrators at $221.210 \mathrm{GHz}$ were instead $0923+392$ and $3 \mathrm{C} \mathrm{84}$, whose fluxes at this frequency are $3.11 \mathrm{Jy}$ and $7.71 \mathrm{Jy}$, respectively. The absolute flux calibration obtained is typically precise to better than $10 \%$ at $110.697 \mathrm{GHz}$ and $\sim 20 \%$ at $221.210 \mathrm{GHz}$ (Castro-Carrizo \& Neri 2010). After calibration, old (2009) and new (2010) data of the CO(1-0) emission line were merged together.

Both the narrow-band and the wide-band (WideX) correlators offered by the PdBI were exploited in our measurements. The WideX provides a spectral resolution of $1.95 \mathrm{MHz}$ over its full bandwidth of $3.6 \mathrm{GHz}$ and is available in parallel to the narrow-band correlator. The narrow-band correlator, whose maximum signal bandwidth is $2 \mathrm{GHz}$, was configured to have a spectral resolution of $2.5 \mathrm{MHz}$. We rebinned the uv-tables obtained with the narrow-band correlator to give frequency channels of $12.5 \mathrm{MHz}$ for $\mathrm{CO}(1-0)$ and $25 \mathrm{MHz}$ for $\mathrm{CO}(2-1)$. The wide-band data, thanks to the larger number of line-free channels available, were exploited to produce uv-tables of the continuum emission (sampled from channels with velocities $|v|>$ $\left.1200 \mathrm{~km} \mathrm{~s}^{-1}\right)$, which was then subtracted from both the $\mathrm{CO}(1-0)$ and $\mathrm{CO}(2-1)$ narrow-band and WideX data.

Data imaging, cleaning, and analysis were performed using the MAPPING software, which is included in the GILDAS package. The field of view of the maps is $2.7 \times 2.7 \mathrm{arcmin}^{2}$ at $110.697 \mathrm{GHz}$ and $1.3 \times 1.3 \operatorname{arcmin}^{2}$ at $221.210 \mathrm{GHz}$. We extracted the $\mathrm{CO}(1-0)$ and $\mathrm{CO}(2-1)$ spectra from the cleaned and continuum-subtracted narrow-band data cubes with an aperture of a diameter of 6 arcsec. The noise levels per channel of the $\mathrm{CO}(1-0)$ and $\mathrm{CO}(2-1)$ spectra are $0.4 \mathrm{mJy}$ and $1.2 \mathrm{mJy}$, respectively. We verified that the noise distribution is Gaussian by analyzing the distribution histogram of the fluxes.

\section{Results}

Figure 1 shows the IRAM PdBI spectra of the $\mathrm{CO}(1-0)$ and $\mathrm{CO}(2-1)$ emission lines extracted from an aperture of 6 arcsec. The broad component, discovered in the (1-0) transition by Feruglio et al. (2010) is also clearly present in the

${ }^{1}$ http://www.iram.fr/IRAMFR/GILDAS 

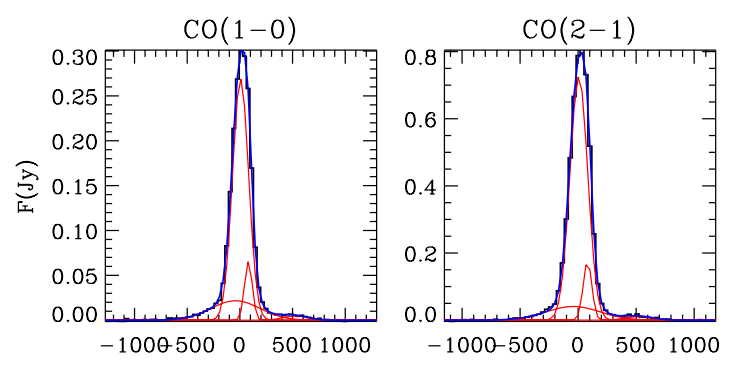

Table 2. Integrated fluxes.

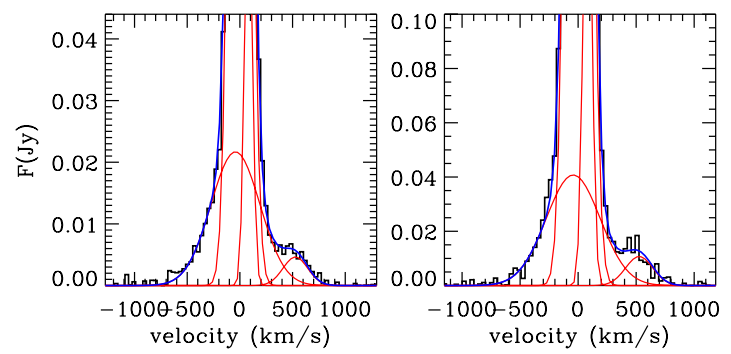

\begin{tabular}{|c|c|c|c|c|}
\hline \multicolumn{5}{|c|}{ Fluxes from uvfits in velocity intervals } \\
\hline & \multicolumn{2}{|c|}{$\begin{array}{c}\text { Velocity range } \\
\left(\mathrm{km} \mathrm{s}^{-1}\right)\end{array}$} & $\begin{array}{c}F[\mathrm{CO}(1-0)] \\
\left(\mathrm{Jy} \mathrm{km} \mathrm{s}{ }^{-1}\right)\end{array}$ & $\begin{array}{l}F[\mathrm{CO}(2-1)] \\
\left(\mathrm{Jy} \mathrm{km} \mathrm{s}^{-1}\right)\end{array}$ \\
\hline Narrow core & \multicolumn{2}{|c|}{$(-300 \div 300)$} & $(64.39 \pm 0.13)$ & $(194.00 \pm 0.50)$ \\
\hline Blue wing & \multicolumn{2}{|c|}{$(-1000 \div-400)$} & $(1.84 \pm 0.16)$ & $(3.90 \pm 0.38)$ \\
\hline Red wing & \multicolumn{2}{|c|}{$(400 \div 1000)$} & $(2.32 \pm 0.17)$ & $(7.08 \pm 0.84)$ \\
\hline Total flux & \multicolumn{2}{|c|}{$(-1000 \div 1000)$} & $(75.98 \pm 0.22)$ & $(241.32 \pm 0.66)$ \\
\hline \multicolumn{5}{|c|}{ Fluxes of Gaussian components } \\
\hline Component & $\begin{array}{l}\text { Velocity } \\
\left(\mathrm{km} \mathrm{s}^{-1}\right)\end{array}$ & $\begin{array}{c}F W H M \\
\left(\mathrm{~km} \mathrm{~s}^{-1}\right)\end{array}$ & $\begin{array}{c}F[\mathrm{CO}(1-0)] \\
\left(\mathrm{Jy} \mathrm{km} \mathrm{s}^{-1}\right)\end{array}$ & $\begin{array}{l}F[\mathrm{CO}(2-1)] \\
\left(\mathrm{Jy} \mathrm{km} \mathrm{s}^{-1}\right)\end{array}$ \\
\hline Narrow core ${ }^{\dagger}$ & $\begin{array}{c}(6.5 \pm 2.0) \\
(80.9 \pm 1.3)\end{array}$ & $\begin{array}{c}(179.7 \pm 2.6) \\
(86.3 \pm 5.3)\end{array}$ & $(54.6 \pm 1.1)$ & $(173.2 \pm 3.3)$ \\
\hline Bluer comp. & $(-42 \pm 16)$ & $(548 \pm 35)$ & $(24.9 \pm 2.5)$ & $(52.7 \pm 6.6)$ \\
\hline Redder comp. & $(527 \pm 30)$ & $(276 \pm 71)$ & $(1.96 \pm 0.43)$ & $(6.1 \pm 1.4)$ \\
\hline & Total flux & & $(75.7 \pm 1.9)$ & $(240.7 \pm 5.6)$ \\
\hline
\end{tabular}

Fig. 1. Continuum-subtracted IRAM PdBI spectra of the $\mathrm{CO}(1-0)$ and $\mathrm{CO}(2-1)$ emission lines. These spectra are extracted from circular apertures with diameters of 6 arcsec. Top panels: full flux scale. Bottom panels: expanded flux scale to highlight the broad components. The two emission lines were simultaneously fitted with four Gaussian functions (red profiles, see text) tied to have the same centers and widths for the two transitions, to reproduce the three main components of each line (i.e. core, blue, and red broad components). The blue contours represent the results of the fit.

Table 1. Description of the IRAM PdBI CO observations.

\begin{tabular}{lccccc}
\hline \hline $\begin{array}{l}\text { Line } \\
\text { (freq.) }\end{array}$ & Date(s) & $\begin{array}{c}\text { Conf. } \\
\text { (no. of ant.) }\end{array}$ & $\begin{array}{c}\text { On source } \\
\text { time }\end{array}$ & $\begin{array}{c}\text { Beam } \\
(\operatorname{arcsec})\end{array}$ & Ref. \\
\hline $\mathrm{CO}(1-0)$ & June-Nov. & $\mathrm{C}+\mathrm{D}$ & $20 \mathrm{~h}$ & $3.2 \times 2.8$ & Feruglio \\
$(110.607 \mathrm{GHz})$ & 09 & $(5$ ant.) & & & et al. $(2010)$ \\
& Oct. 10 & $\begin{array}{c}\mathrm{C} \\
(5 / 6 \text { ant.) }\end{array}$ & $7.4 \mathrm{~h}$ & & This work \\
& & & & \\
\hline $\mathrm{CO}(2-1)$ & Sep.-Nov. & C+D & $4.2 \mathrm{~h}$ & $1.6 \times 1.3$ & This work \\
$(221.210 \mathrm{GHz})$ & 10 & $(5 / 6$ ant.) & & & \\
\hline
\end{tabular}

(2-1) transition. The spectra in Fig. 1 are fitted by using two narrow Gaussians to fit the core of the $\mathrm{CO}$ lines and two broad Gaussians to fit the wings. In the fitting, the centers and widths of the components are constrained to be identical for the two $\mathrm{CO}$ transitions, but their relative strengths are allowed to vary. The need to use two narrow Gaussians to fit the core probably reflects that the gas distribution in the central rotating disk cannot be described by a simple, single Gaussian.

The fluxes extracted from the cleaned cubes may be affected by various uncertainties associated with the cleaning process and aperture effects. Therefore, to estimate rigorously the fluxes of the components, we used the fluxes inferred directly from the visibility data, which are unaffected by any cleaning problem. More specifically, we measured the flux inferred from the uv amplitudes at the $25 \mathrm{~m}$ baseline, for which the beam width is 14 arcsec at $230 \mathrm{GHz}$, and is even larger at lower frequencies: this therefore ensures that all of the flux in the wings (which are much less extended, as discussed below) is accounted for. To obtain this information, we extracted, for both the $\mathrm{CO}$ transitions, the amplitude uv diagrams of each spectral line component separately, by averaging the frequency channels corresponding to $-300 \lesssim v\left(\mathrm{~km} \mathrm{~s}^{-1}\right) \lesssim 300$ (line core), $-1000 \lesssim v\left(\mathrm{~km} \mathrm{~s}^{-1}\right) \lesssim$ -400 (blue wing), and $400 \lesssim v\left(\mathrm{~km} \mathrm{~s}^{-1}\right) \lesssim 1000$ (red wing). All

Notes. A flux calibration systematic error equal to the $10 \%$ of the flux value should be added in quadrature to the statistical error reported with each flux measurement in this table. ${ }^{(\dagger)}$ The FWHM and the velocity of both the Gaussians fitting the narrow core are reported.

three components were continuum-subtracted. In addition, for the visibilities of the narrow core, we also subtracted the contribution of the "near" wings estimated within $-600 \lesssim v\left(\mathrm{~km} \mathrm{~s}^{-1}\right) \lessgtr$ -400 and $400 \lesssim v\left(\mathrm{~km} \mathrm{~s}^{-1}\right) \lesssim 600$. In Table 2, we report the fluxes of the $\mathrm{CO}$ lines, integrated within these velocity intervals in the uv-amplitude data, along with the total line fluxes estimated within $-1000 \lesssim v\left(\mathrm{~km} \mathrm{~s}^{-1}\right) \lesssim 1000$, by applying the same method $^{2}$. The wings are detected at a confidence level ranging from $8 \sigma$ to $14 \sigma$, depending on the transition.

The full width at half maximums (FWHMs), velocities, and fluxes of the Gaussian components resulting from the fit shown in Fig. 1, are also reported in Table 2. The fluxes of the Gaussian components were corrected for aperture and cleaning effects by rescaling their fluxes to the values obtained from the uv-amplitudes in the following way: we integrated the spectra shown in Fig. 1 in the same velocity intervals as those used for the uv-amplitudes of the narrow core and the broad wings, and then estimated the three different scaling factors from the ratios of the fluxes resulting from the uv-amplitudes to the fluxes obtained by integrating the spectra of the individual Gaussian components. We note that the scaling factors obtained for the broad wings are slightly higher than those obtained for the narrow core, since the spectra extracted from an aperture with a diameter of only 6 arcsec miss part of the broad and faint components of the two $\mathrm{CO}$ emission lines.

Figures 2 and 3 show the maps of the blue and red wings of the $\mathrm{CO}(1-0)$ and $\mathrm{CO}(2-1)$ emission lines and the half-power contours of the synthesized beams. The emission from both the broad components of the $\mathrm{CO}(1-0)$ and $\mathrm{CO}(2-1)$ lines is spatially resolved. The peaks of the red and blue wings of both the $\mathrm{CO}(1-0)$ and the $\mathrm{CO}(2-1)$ emission lines are not spatially offset from one another. This validates the hypothesis that these highvelocity components are due to the motion of outflowing gas,

\footnotetext{
${ }^{2}$ Note that the errors reported in Table 2 are statistical errors only. These are the ones required to infer the statistical significance and compare the relative intensity of the various components within the same transition. However, when comparing with other observations or among different transitions, a systematic error of $10 \%$ in the absolute calibration should be added in quadrature.
} 

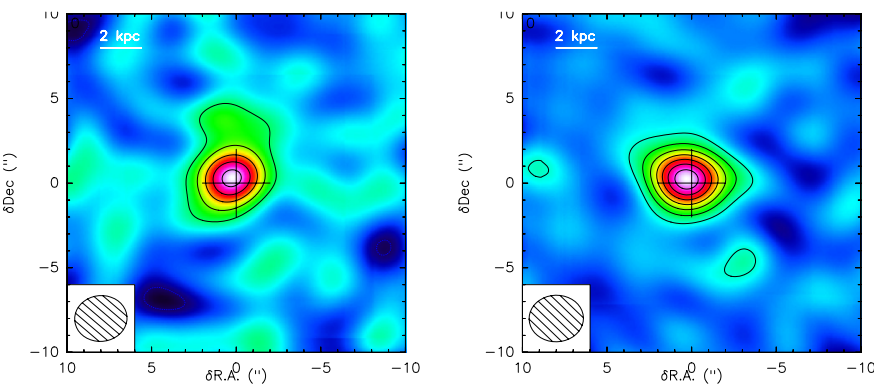

Fig. 2. IRAM PdBI maps of the $\mathrm{CO}(1-0)$ blue (left panel) and red (right panel) broad wings. The size of the map is $20 \times 20$ arcsec. Contours correspond to $3 \sigma\left(1 \sigma=0.14 \mathrm{mJy}_{\text {beam }}{ }^{-1}\right)$. The synthesized beam size is shown at the bottom of the maps. The cross indicates the peak of the radio (VLBI) emission. Note that the peaks of the wing maps appear to be slightly offset from the peak of the radio VLBI but are well within the beam.
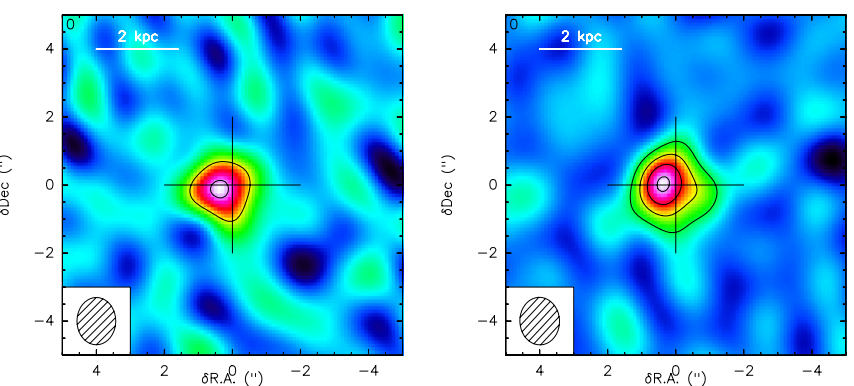

Fig. 3. IRAM PdBI maps of the $\mathrm{CO}(2-1)$ blue (left panel) and red (right panel) broad wings. The size of the map is $10 \times 10$ arcsec. Contours correspond to $3 \sigma\left(1 \sigma=0.6 \mathrm{mJy}^{\text {beam }}{ }^{-1}\right)$. The synthesized beam size is shown at the bottom of the maps. The cross indicates the peak of the radio (VLBI) emission. A tapering with uv taper $=50 \mathrm{~m}$ has been applied to both the maps; the tapering cuts the visibilities with uv radius $<50 \mathrm{~m}$, so the maps appear more detailed and the synthesized beam is slightly smaller than in the original maps. Note that the peaks of the wing maps appear to be slightly offset from the peak of the radio VLBI but are well within the beam.

which moves in roughly an axisymmetric way relative to the line of sight, instead of a rotating molecular disk. That a large fraction of the outflow occurs along our line of sight is supported by the blueshifted $\mathrm{OH}$ absorption observed in a Herschel PACS spectrum (Fischer et al. 2010).

To quantify the extension of the wings, in Fig. 4 we plot the amplitude of the visibilities as a function of the uv radius for both the blue and red wings of the $\mathrm{CO}(1-0)$ and $\mathrm{CO}(2-1)$ emission lines. We have binned the visibilities in baseline steps of $50 \mathrm{~m}$. The data points in Fig. 4 were fitted with both a point (unresolved) source model and a circular Gaussian source model. The resulting reduced $\chi^{2}$ and the associated probability $P$ values of the best fits are reported in Table 3, which also gives the differences $\Delta \chi^{2}=\chi^{2}$ (point)- $\chi^{2}$ (Gaussian) and their associated $1-P$. We estimated the physical extent of the outflow in terms of the FWHM of the best-fit circular Gaussian model fits for the uv data. It should be noted that since we directly fitted the uv data, the fit automatically takes into account the interferometric beam size. The resulting sizes are reported in Table 3. We note that while the uvfit is generally good, in some cases (at some uv radii), the Gaussian fit to the amplitudes deviates by about $1.5 \sigma$ from the observed values, this means that the spatial distribution of the flux is probably more complex than the simple circular Gaussian approximation assumed by us.
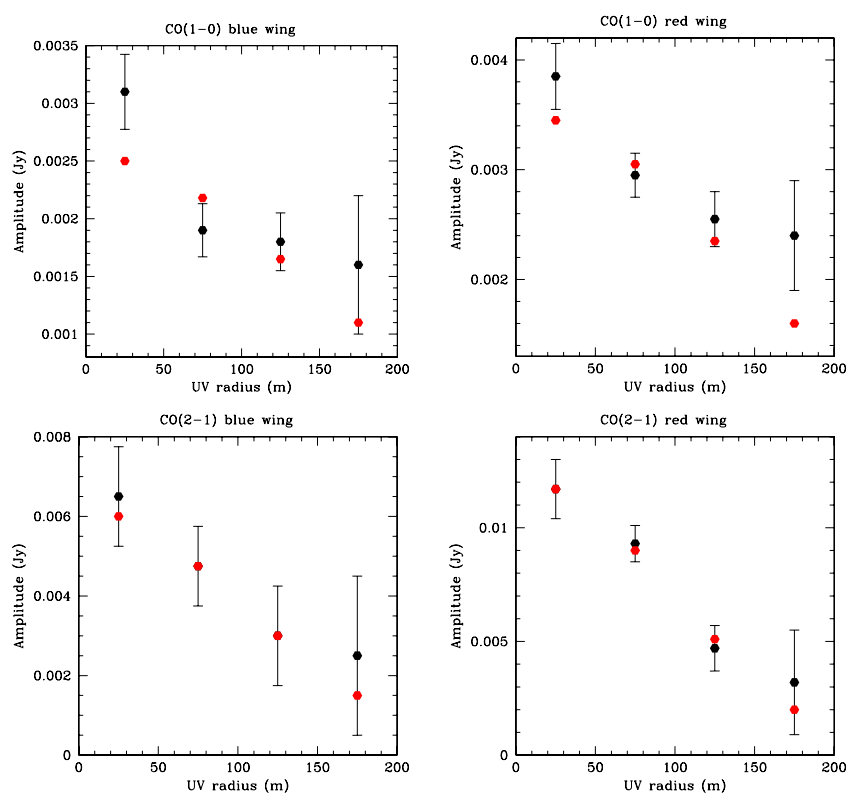

Fig. 4. Amplitude of visibilities (Jy) plotted as a function of the uv radius for the blue and red wings of the $\mathrm{CO}(1-0)$ and $\mathrm{CO}(2-1)$ emission lines (black points with errorbars). The visibilities were binned using intervals of $50 \mathrm{~m}$ of uv radius, covering baselines from $10 \mathrm{~m}$ to $200 \mathrm{~m}$. The emission from the wings was estimated within the velocity intervals $-1000 \lesssim v\left(\mathrm{~km} \mathrm{~s}^{-1}\right) \lesssim-400$ and $400 \lesssim v\left(\mathrm{~km} \mathrm{~s}^{-1}\right) \lesssim 1000$. We recall that for a point (i.e. unresolved) source the visibility amplitude is a constant function of the uv radius. The red points are the results of the best fits to a circular Gaussian model.

The results in Table 3 demonstrate that both the red and blue wings of the $\mathrm{CO}(1-0)$ and those of the $\mathrm{CO}(2-1)$ lines are spatially resolved at high levels of significance (much higher than previously reported in Feruglio et al. 2010, for the $\mathrm{CO}(1-0)$ thanks to the stronger signal on long baselines even for this transition). More specifically, the spatial distribution of the wings is resolved with a confidence ranging from $5 \sigma$ to $11 \sigma$, depending on the transition and the wing component. These results prove that the outflow extends out to the kpc scale: $F W H M \sim 1.2 \mathrm{kpc}$ in the case of the $\mathrm{CO}(1-0)$ transition and $F W H M \sim 0.8 \mathrm{kpc}$ for the $\mathrm{CO}(2-1)$ transition.

Summarizing, our new data not only detect for the first time the broad wings of the $\mathrm{CO}(2-1)$ transition, and resolve them spatially, but also allow us to estimate the extension of the $\mathrm{CO}(1-0)$ broad wings with much higher accuracy than previous studies (Feruglio et al. 2010).

\section{Discussion}

\subsection{The $\mathrm{CO}(2-1) / C O(1-0)$ outflow excitation ratio}

The physical conditions of molecular gas in galaxies, such as its kinetic temperature, volume density, and optical depth, can be derived from the $\mathrm{CO}$ excitation properties and, in particular, from the relative strengths of the $\mathrm{CO}$ emission lines. The physical conditions of the molecular gas responsible for the narrow components of Mrk 231's CO emission lines have been widely investigated. Papadopoulos et al. (2007) and van der Werf et al. (2010) found that the rotational excitation diagram of the narrow core of Mrk 231's CO lines is dominated, in the lower $J$ levels $\left(J_{\text {upper }} \in[1,3]\right)$, by a component of density $n\left(\mathrm{H}_{2}\right) \sim 10^{3} \mathrm{~cm}^{-3}$ and excitation temperature $T \in[55,95] \mathrm{K}$. In principle, a similar analysis could be undertaken for the $\mathrm{CO}$ wings tracing the 
Table 3. Best-fit results of the visibility versus uv radius plots.

\begin{tabular}{lcccccc}
\hline \hline Line & $\begin{array}{c}\text { Velocity range } \\
\left(\mathrm{km} \mathrm{s}^{-1}\right)\end{array}$ & $\begin{array}{c}\text { Point } \\
\text { source model }\end{array}$ & $\begin{array}{c}\text { Gaussian } \\
\text { source model }\end{array}$ & $\Delta \chi^{2 \dagger}$ & $\begin{array}{c}F W H M \\
(\mathrm{arcsec})\end{array}$ & $\begin{array}{c}F W H M^{\ddagger} \\
(\mathrm{kpc})\end{array}$ \\
\hline $\mathrm{CO}(1-0)$ & {$[-1000,-400]$} & $\chi_{r}^{2}=4.14$ & $\chi_{r}^{2}=1.98$ & $\Delta \chi^{2}=6.48$ & $(1.52 \pm 0.31)$ & $(1.26 \pm 0.25)$ \\
blue wing & & $P=6.1 \mathrm{E}-03$ & $P=0.11$ & $(1-P)=0.99$ & & \\
\hline $\mathrm{CO}(1-0)$ & {$[400,1000]$} & $\chi_{r}^{2}=4.26$ & $\chi_{r}^{2}=1.74$ & $\Delta \chi^{2}=7.54$ & $(1.37 \pm 0.22)$ & $(1.13 \pm 0.18)$ \\
red wing & & $P=5.2 \mathrm{E}-03$ & $P=0.16$ & $(1-P)=0.99$ & & \\
\hline $\mathrm{CO}(2-1)$ & {$[-1000,-400]$} & $\chi_{r}^{2}=1.69$ & $\chi_{r}^{2}=0.14$ & $\Delta \chi^{2}=4.65$ & $(0.80 \pm 0.16)$ & $(0.66 \pm 0.14)$ \\
blue wing & & $P=0.17$ & $P=0.94$ & $(1-P)=0.97$ & & \\
\hline $\mathrm{CO}(2-1)$ & {$[400,1000]$} & $\chi_{r}^{2}=8.94$ & $\chi_{r}^{2}=0.19$ & $\Delta \chi^{2}=26.24$ & $(1.01 \pm 0.09)$ & $(0.84 \pm 0.07)$ \\
red wing & & $P=6.5 \mathrm{E}-06$ & $P=0.90$ & $(1-P) \simeq 1.00$ & & \\
\hline
\end{tabular}

Notes. ${ }^{(\dagger)} \Delta \chi^{2}=\chi^{2}$ (point)- $\chi^{2}$ (Gaussian). ${ }^{(\ddagger)}$ The adopted cosmology and redshift result in a spatial scale of $0.828 \mathrm{kpc}^{\operatorname{arcsec}^{-1}}$.

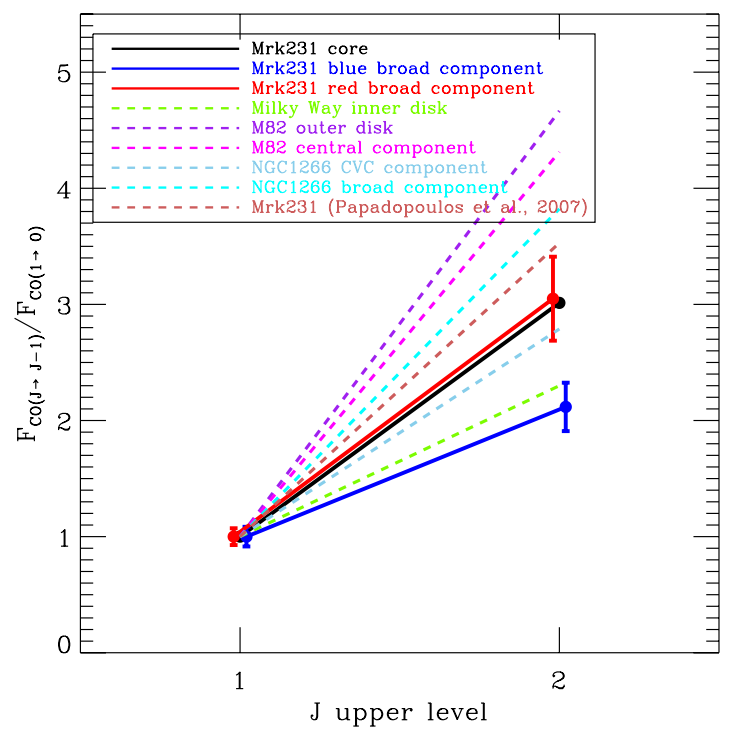

Fig. 5. Relative strengths of both the narrow core (black) and the broad wings (blue and red) of Mrk 231's CO emission lines. The ratios of the $\mathrm{CO}$ integrated fluxes were calculated using the data reported in the upper part of Table 2. The CO spectral line energy distribution (SLED) of Mrk 231 obtained by Papadopoulos et al. (2007) is shown for comparison, along with the CO SLEDs of the Milky Way (Fixsen et al. 1999), M 82 (Weiß et al. 2005) and NGC 1266 (Alatalo et al. 2011).

outflow. However, two CO transitions are certainly not enough to investigate the excitation and physical conditions of the outflowing gas. As a consequence, we simply compare in the following the $\mathrm{CO}(1-0) / \mathrm{CO}(2-1)$ ratio observed in the wings with the same ratio observed in the core of the line and with other environments.

Figure 5 shows the $\mathrm{CO}(1-0) / \mathrm{CO}(2-1)$ ratio for the two wings and for the line core, compared with CO SLEDs observed in other galaxies, more specifically: the Milky Way's inner disk $(2.5<|l|<32.5)$ (Fixsen et al. 1999), the outer disk and the central component of M 82 (Weiß et al. 2005), both the core and the broad component of NGC 1266 (Alatalo et al. 2011), and the CO core SLED of Mrk 231 obtained by Papadopoulos et al. (2007). It should be noted that the error bars in Fig. 5 represent only the statistical uncertainties, since here we are primarily interested in the excitation of the wings relative to the core (hence the absolute calibration uncertainties are unimportant). However, when comparing excitation ratios with other targets, an uncertainty of $10 \%$ should be added in quadrature.

The $\mathrm{CO}(1-0) / \mathrm{CO}(2-1)$ ratios observed in the red wing and in the narrow core are consistent with each other. The blue wing is less excited than the core of the line. Taken at face value, this result does not support AGN feedback models where the outflow is generated by a radiation-driven shock ("blast wave models"), which, besides accelerating the ISM, should greatly increase its temperature and density (e.g. Lapi et al. 2005; Zubovas \& King 2012). However, it is possible that a high excitation component of the outflow may be revealed by high-J transitions. Therefore, sensitive and wide band observations of higher $\mathrm{CO}$ transitions are required to further investigate this issue.

However, it should be noted that, since the $\mathrm{CO}(2-1)$ wings are more compact than the $\mathrm{CO}(1-0)$ wings, the $\mathrm{CO}(2-1) / \mathrm{CO}(1-0)$ ratio is likely to vary radially. More specifically, the $\mathrm{CO}(2-1) / \mathrm{CO}(1-0)$ ratio should be higher in the central region than the outer region. Higher angular resolution maps would be required to properly map the $\mathrm{CO}(2-1) / \mathrm{CO}(1-0)$ ratio and disentangle the beam-smearing effects. However, on the basis of the circular Gaussian modeling of the spatial distribution of the two wings in the two transitions, we estimated that within the central $0.7 \mathrm{kpc}$ (FWHM of the blue wing) the $\mathrm{CO}(2-1) / \mathrm{CO}(1-0)$ ratio of the blue wing increases to $3.5 \pm 0.8$, which is however still consistent with the excitation observed in the core of the lines $\left(F_{\mathrm{CO}(2-1)} / F_{\mathrm{CO}(1-0)}=2.9\right)$. The $\mathrm{CO}(2-1) / \mathrm{CO}(1-0)$ flux ratio of the red wing increases in the same inner region to $4.0 \pm 0.8$, which starts to be inconsistent with the excitation in the core of the line, although marginally. On the other hand, in the region outside the central $0.7 \mathrm{kpc}$ the $\mathrm{CO}(2-1) / \mathrm{CO}(1-0)$ ratio drops significantly, below 2 , well below the excitation observed in the core of the line. Summarizing, the outflowing gas associated with the blue wing does not show any evidence of higher excitation relative to the core of the line, both globally and even in the central region, indicating that this outflowing gas is unaffected by shocks. Within the central region, the gas associated with the red wing does show marginally higher excitation, suggesting that this component of the outflowing gas may be affected by some shocks.

The high Mach number inferred by Feruglio et al. (2010), based on the outflow velocity of the molecular clouds, is also indicative of shocks. However, we also note that high outflow velocities do not necessarily translate into a large Mach number, since the medium in which the clouds are embedded may be outflowing with the same velocity (as appears to be the case from the similar velocity of the ionized gas found by Rupke \& Veilleux 2011), hence resulting in a smaller differential velocity between the molecular clouds and the surrounding gas.

Another important implication of our result is that the CO-to- $\mathrm{H}_{2}$ conversion factor in the wings may not differ too much from the bulk of the gas in the host galaxy traced by the 


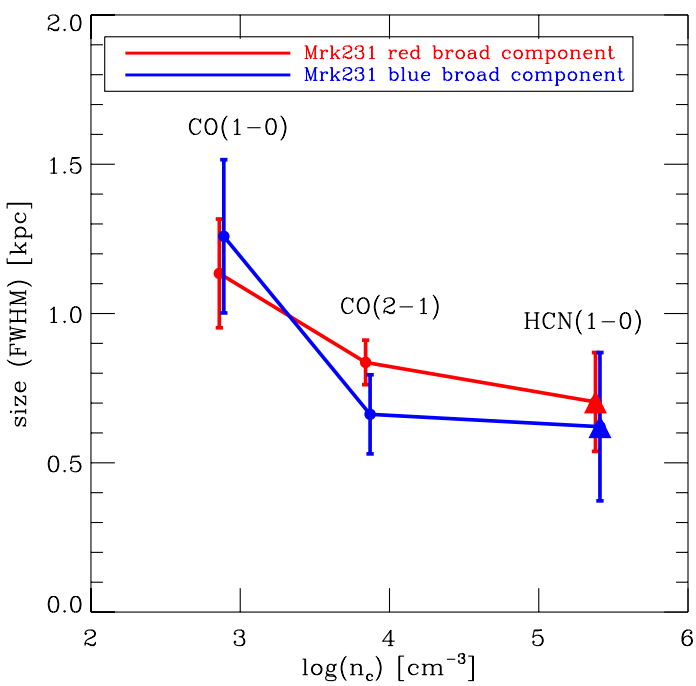

Fig. 6. Size (FWHM) of the outflow plotted as a function of the critical density of the corresponding molecular transition used as a wind tracer. Our $\mathrm{CO}(1-0)$ and $\mathrm{CO}(2-1)$ IRAM PdBI data are represented by filled circles, while the HCN(1-0) observations by Aalto et al. (2012) are denoted by filled triangles. Critical densities were calculated for a molecular medium at $T=100 \mathrm{~K}$. Note that the same conversion factor of $0.828 \mathrm{kpc} \operatorname{arcsec}^{-1}$ has been applied to all data in order to allow comparisons.

narrow CO component (i.e. a ULIRG-like conversion factor). In Feruglio et al. (2010), we had been conservative by assuming, for the outflow, a conversion factor $\alpha=0.5 M_{\odot}\left(\mathrm{K} \mathrm{km} \mathrm{s}^{-1} \mathrm{pc}^{2}\right)^{-1}$ (i.e. one-tenth of the Galactic value and $40 \%$ lower than the value assumed for ULIRGs); this is the lowest conversion factor found in different locations of M 82, including its molecular outflow (Weiß et al. 2001). The finding that the CO excitation in the outflow does not differ significantly from (or is even lower) than the core of the line, tracing the bulk of the gas in this ULIRG, suggests that the conversion factor is also similar (i.e. $\left.\alpha=0.8 M_{\odot}\left(\mathrm{K} \mathrm{km} \mathrm{s}^{-1} \mathrm{pc}^{2}\right)^{-1}\right)$. This implies that the outflow molecular mass and outflow rate given in Feruglio et al. (2010) are underestimated, and are probably higher by a factor of about 1.4 .

We note that in the jet-driven outflow observed in NGC 1266 by Alatalo et al. (2011) the excitation of the CO broad components tends to be higher than in the central velocity component (as illustrated in Fig. 5), although not strongly. This is not unexpected. In this case, the outflow is totally driven by jet-induced shocks, which unavoidably heat the ISM and therefore produce higher $\mathrm{CO}$ excitation. The outflow $\mathrm{CO}$ excitation differences between Mrk 231 and NGC 1266 further highlight that the driving mechanism in the two sources are different, although both associated with an AGN.

Finally, we note that the Mrk 231 SLED of the core obtained by Papadopoulos et al. (2007) by means of single dish observations is slightly steeper than the one obtained by us. We ascribe the difference partly to calibration uncertainties and partly to uncertainties in subtracting the continuum and broad wings with previous narrow band spectra (especially in single dish data where the baseline instabilities may be problematic).

\subsection{Extension of the outflow}

As mentioned above, our new data have allowed us to constrain the size of the molecular outflow traced by the $\mathrm{CO}$ transitions with unprecedented accuracy, enabling analyses that have not been possible until now. The results presented in the previous section clearly reveal that the extension of the outflow traced by the $\mathrm{CO}(1-0)$ transition is significantly larger than observed in the $\mathrm{CO}(2-1)$ and $\mathrm{HCN}(1-0)$ transitions. Figure 6 shows the extension of the outflow as a function of the critical density of the transition used to trace the outflow, where the red and blue symbols indicate the trends relative to the red and blue wings, respectively. There is a clear trend for the size of the outflow to decrease as a function of the critical density, at least in the range $10^{3}<n_{\mathrm{cr}}<10^{4} \mathrm{~cm}^{-3}$ (while at higher densities it is more difficult to assess this trend owing to the large errorbars in the size of the HCN wings).

A radial decreasing density of the outflowing molecular gas is also consistent with the Herschel measurements by Fischer et al. (2010) and Sturm et al. (2011), and in particular with the blueshifted absorption profile of the far-IR molecular transitions observed in the PACS spectra (OH $79 \mu \mathrm{m}, \mathrm{OH} 119 \mu \mathrm{m}$, $\left.{ }^{18} \mathrm{OH} 120 \mu, \mathrm{H}_{2} \mathrm{O} 79 \mu \mathrm{m}\right)$, as discussed in those papers.

This result is consistent with the prediction of Narayanan et al. (2008), who studied the response of CO morphology to starburst and AGN feedback-driven winds in galaxy mergers. According to them, the emission from higher $\mathrm{CO}$ transitions (e.g. $\mathrm{CO}(2-1))$ broadly follows the distribution of the $\mathrm{CO}(1-0)$ emission, but remains slightly more compact. This is because, according to their model, denser clouds (preferentially traced by higher transitions) have shorter lifetimes along the outflow and tend to evaporate into a more diffuse molecular phase, traced by the $\mathrm{CO}(1-0)$ transition.

The result could also possibly support the scenario proposed by Hopkins \& Elvis (2010), where dense clouds invested by an even mild AGN-driven wind develop Kelvin-Helmholtz instabilities causing them to expand and accelerate more easily by means of radiation pressure. In the latter scenario, one would expect diffuse clouds to be driven to larger distances than the denser clouds. However, Kelvin-Helmholtz instabilities should also develop shocks (although much milder than those expected in "blast wave models") that should show up in higher gas excitation, which is not the case for the blue wing, but may be for the red wing.

Simple acceleration by radiation pressure on the dust in molecular clouds (Fabian et al. 2009), without invoking any scenario associated with shocks, may not only explain the modest $\mathrm{CO}$ excitation in the wings, but also the size difference between the $\mathrm{CO}(1-0)$ and $\mathrm{CO}(2-1)+\mathrm{HCN}$ emission components. More diffuse clouds (preferentially traced by $\mathrm{CO}(1-0)$ ) are indeed characterized by a higher ratio between the outward radiation pressure and inward gravitational pull than dense clouds, which are preferentially traced by $\mathrm{CO}(2-1)+\mathrm{HCN}$ emission. More specifically, for clouds whose dust is optically thick to $\mathrm{UV} /$ optical radiation, the ratio $F_{\text {rad }} / F_{\text {grav }}$ is inversely proportional to the column density of the cloud $N_{\mathrm{H}}$, which is higher in dense clouds. As a consequence, diffuse clouds can be expelled to larger distances. Radiation pressure on the dust in molecular clouds can in principle also develop shocks, but this depends on whether the medium in which the molecular clouds are embedded is comoving with them.

We have discussed so far only the global size of the emitting regions of the two $\mathrm{CO}$ transitions obtained by fitting the uv data with a simple circular Gaussian. At our resolution, the maps do not show much structure. The only relevant structure at the $3 \sigma$ level is possibly an extension of the $\mathrm{CO}(1-0)$ blue wing (Fig. 2, left) a few arcseconds to the north. This could imply that there is an association between some of the molecular outflow (a minor part of it) and the northern region, where the radio jet 
affects the neutral gas outflow as inferred by Rupke \& Veilleux (2011) from the blue-shifted Na I D kinematics map. Aalto et al. (2012) detected a plum extending to the north in the HCN(1-0) red-shifted wing map and also inferred a link between this feature and the jet-influenced neutral wind detected by Rupke \& Veilleux (2011). However, we propose that this association is incorrect, since the jet-accelerated gas is detected in terms of blueshifted $\mathrm{Na}$ I $\mathrm{D}$ absorption, so any association should be seen in the molecular blue wings, not the red wings. The possible interaction between part of the molecular outflow and the radio-jet should however be investigated using higher-resolution data.

Finally, we note that the size ( $\sim 2$ arcsec) of the (blueshifted) "nuclear wind", which is the region identified by Rupke \& Veilleux (2011) where the outflow is dominated by a radiationpressure drive wind, is close to the size $(1.5 \pm 0.3 \mathrm{arcsec})$ of the molecular outflow determined by us from the blue wing of the $\mathrm{CO}(1-0)$ component.

\section{Conclusions}

We have presented new broad-band IRAM PdBI observations of the $\mathrm{CO}(2-1)$ transition in Mrk 231, which is the ULIRG hosting the closest quasar known. For the first time, our observations reveal the broad wings of the $(2-1)$ transition extending to velocities of $F W Z I \sim 1500 \mathrm{~km} \mathrm{~s}^{-1}$, which trace the same molecular wind revealed by previous $\mathrm{CO}(1-0) \mathrm{PdBI}$ observations and PACS far-IR spectroscopy of $\mathrm{OH}$ transitions. The $\mathrm{CO}(2-1)$ wings are spatially resolved, with an extension of about $0.8 \mathrm{kpc}$.

We have also obtained new $\mathrm{CO}(1-0)$ observations, by significantly improving the signal at high angular resolution relative to previous works, which allows us to determine more accurately the size of the molecular outflow traced by this transition. The $\mathrm{CO}(1-0)$ broad wings are spatially resolved with a significance that is much higher $(5-7 \sigma)$ than in previous observations. We measure an extension of the $\mathrm{CO}(1-0)$ wings of about $1.2 \mathrm{kpc}$, which is significantly larger than observed for the $\mathrm{CO}(2-1)$ wings. The extension of the $\mathrm{CO}(1-0)$ wings is also significantly larger than the extension of the $\mathrm{HCN}(1-0)$ wings, tracing high density gas in the outflow detected by previous observations. More specifically, we show that the size of the outflow anticorrelates with the critical density of the transitions used to trace the outflow.

These results are consistent with the scenario where denser clouds (traced by the $\mathrm{CO}(2-1)$ and $\mathrm{HCN}(1-0)$ broad wings) have shorter lifetimes along the outflow and evaporate into a more diffuse molecular component (traced by $\mathrm{CO}(1-0)$ ) at larger radii, as predicted by some feedback models.

We also find that the $\mathrm{CO}$ excitation in the outflow, as traced by the $\mathrm{CO}(2-1) / \mathrm{CO}(1-0)$ ratio of the broad wings, does not differ significantly from that of the gas in the bulk of the galaxy, as traced by the core of the CO lines. In the blue wing, the excitation is lower than in the core of the line. Taken at face value, this result in inconsistent with models where the molecular outflow is driven by a shock wave generated by the interaction of a radiation pressure-driven nuclear wind with the ISM of the host galaxy. We favor a scenario where gas clouds are directly accelerated by the radiation pressure on dust. This scenario does not need to invoke shocks, hence the $\mathrm{CO}$ excitation is unaffected. Moreover, in this scenario low density clouds (traced by $\mathrm{CO}(1-0))$ are accelerated more efficiently, hence reaching larger distances than dense clouds (traced by $\mathrm{CO}(2-1)$ and $\mathrm{HCN}$ ). However, we also note that in the inner region $(R<0.3 \mathrm{kpc})$, the $\mathrm{CO}(2-1) / \mathrm{CO}(1-0)$ ratio is indeed slightly higher, which is indicative of some shock contribution.
We marginally detect an extension of the $\mathrm{CO}(1-0)$ blueshifted wing to the north, where previous studies have found evidence that the atomic neutral outflow, traced by blueshifted $\mathrm{Na}$ I D absorption, is influenced by the radio jet. This suggests that the radio jet may also contribute to the acceleration of part of the molecular outflow, although far less significantly than the global molecular outflow.

Acknowledgements. We thank Andrea Lapi for helpful suggestions and discussions. We are grateful to the IRAM staff in Grenoble for helping with data reduction and calibration. This work is based on observations carried out with the IRAM Plateau de Bure Interferometer. IRAM is supported by INSU/CNRS (France), MPG (Germany), and IGN (Spain). This work was supported by ASI/INAF contract I/009/10/0.

\section{References}

Alatalo, K., Blitz, L., Young, L. M., et al. 2011, ApJ, 735, 88

Aalto, S., Garcia-Burillo, S., Muller, S., et al. 2012, A\&A, 537, A44

Alexander, D. M., Swinbank, A. M., Smail, I., McDermid, R., \& Nesvadba, N. P. H. 2010, MNRAS, 402, 2211

Baldry, I. K., Balogh, M. L., Bower, R., Glazebrook, K., \& Nichol, R. C. 2004, in The New Cosmology: Conference on Strings and Cosmology, ed. R. E. Allen, D. V. Nanopoulos, \& C. N. Pope, AIP Conf. Ser., 743, 106

Boroson, T. A., \& Meyers, K. A. 1992, ApJ, 397, 442

Braito, V., Della Ceca, R., Piconcelli, E., et al. 2004, A\&A, 420, 79

Cano-Díaz, M., Maiolino, R., Marconi, A., et al. 2012, A\&A, 537, L8

Castro-Carrizo, A., \& Neri, R. 2010, IRAM Plateau de Bure Interferometer Data Reduction Cookbook

Cattaneo, A., Faber, S. M., Binney, J., et al. 2009, Nature, 460, 213

Croton, D. J., Springel, V., White, S. D. M., et al. 2006, MNRAS, 365, 11

Davies, R. I., Tacconi, L. J., \& Genzel, R. 2004, ApJ, 613, 781

Di Matteo, T., Springel, V., \& Hernquist, L. 2005, Nature, 433, 604

Elvis, M. 2006, Mem. Soc. Astron. It., 77, 573

Fabian, A. C. 1999, MNRAS, 308, L39

Fabian, A. C. 2012, ARA\&A, in press [arXiv: 1204.4114]

Fabian, A. C., Celotti, A., \& Erlund, M. C. 2006, MNRAS, 373, L16

Fabian, A. C., Vasudevan, R. V., Mushotzky, R. F., Winter, L. M., \& Reynolds, C. S. 2009, MNRAS, 394, L89

Farrah, D., Urrutia, T., Lacy, M., et al. 2012, ApJ, 745, 178

Ferrarese, L., \& Ford, H. 2005, Space Sci. Rev., 116, 523

Feruglio, C., Maiolino, R., Piconcelli, E., et al. 2010, A\&A, 518, L155

Fischer, J., Sturm, E., González-Alfonso, E., et al. 2010, A\&A, 518, L41

Fixsen, D. J., Bennett, C. L., \& Mather, J. C. 1999, ApJ, 526, 207

Granato, G. L., De Zotti, G., Silva, L., Bressan, A., \& Danese, L. 2004, ApJ, 600,580

Greene, J. E., Zakamska, N. L., Ho, L. C., \& Barth, A. J. 2011, ApJ, 732, 9

Gültekin, K., Richstone, D. O., Gebhardt, K., et al. 2009, ApJ, 698, 198

Hopkins, P. F., \& Elvis, M. 2010, MNRAS, 401, 7

Hopkins, P. F., Cox, T. J., Kereš, D., \& Hernquist, L. 2008, ApJS, 175, 390

King, A. 2005, ApJ, 635, L121

King, A. R. 2010, MNRAS, 402, 1516

King, A. R., \& Pounds, K. A. 2003, MNRAS, 345, 657

Lapi, A., Cavaliere, A., \& Menci, N. 2005, ApJ, 619, 60

Lipari, S., Sanchez, S. F., Bergmann, M., et al. 2009, MNRAS, 392, 1295

Maiolino, R., Gallerani, S., Neri, R., et al. 2012, MNRAS Lett., submitted [arXiv: 1204.2904]

Marconi, A., \& Hunt, L. K. 2003, ApJ, 589, L21

Menci, N., Fiore, F., Puccetti, S., \& Cavaliere, A. 2008, ApJ, 686, 219

Menci, N., Fontana, A., Giallongo, E., Grazian, A., \& Salimbeni, S. 2006, ApJ, 647,753

Narayanan, D., Cox, T. J., Kelly, B., et al. 2008, ApJS, 176, 331

Nesvadba, N. P. H., Boulanger, F., Salomé, P., et al. 2010, A\&A, 521, A65

Nesvadba, N. P. H., Polletta, M., Lehnert, M. D., et al. 2011, MNRAS, 415, 2359

Papadopoulos, P. P., Isaak, K. G., \& van der Werf, P. P. 2007, ApJ, 668, 815

Rupke, D. S. N., \& Veilleux, S. 2011, ApJ, 729, L27

Sanders, D. B., Mazzarella, J. M., Kim, D.-C., Surace, J. A., \& Soifer, B. T. 2003, ApJ, 126, 1607

Silk, J., \& Rees, M. J. 1998, A\&A, 331, L1

Sturm, E., González-Alfonso, E., Veilleux, S., et al. 2011, ApJ, 733, L16

Taylor, G. B., Silver, C. S., Ulvestad, J. S., \& Carilli, C. L. 1999, ApJ, 519, 185

van der Werf, P. P., Isaak, K. G., Meijerink, R., et al. 2010, A\&A, 518, L42

Weiß, A., Neininger, N., Hüttemeister, S., \& Klein, U. 2001, A\&A, 365, 571

Weiß, A., Walter, F., \& Scoville, N. Z. 2005, A\&A, 438, 533

Zubovas, K., \& King, A. 2012, ApJ, 745, L34 